УДК 517.9

\title{
On Local Solvability of the System of the Equations of One Dimensional Motion of Magma
}

\author{
Alexander A. Papin* \\ Margarita A. Tokareva ${ }^{\dagger}$ \\ Altai State University \\ Lenina, 61, Barnaul, 656049
}

Russia

Received 01.12.2016, received in revised form 20.01.2017, accepted 20.05.2017

The local solvability of initial-boundary value problem for the system of the equations of non stationary motion of magma is proved.

Keywords: Darcy law, poroelastisity, magma, solvability, uniqueness.

DOI: 10.17516/1997-1397-2017-10-3-385-395.

\section{Problem statement. Formulation of main results}

A quasi-linear system of equations of composite type is considered:

$$
\begin{gathered}
\frac{\partial(1-\phi) \rho_{s}}{\partial t}+\frac{\partial}{\partial x}\left((1-\phi) \rho_{s} v_{s}\right)=0, \quad \frac{\partial\left(\rho_{f} \phi\right)}{\partial t}+\frac{\partial}{\partial x}\left(\rho_{f} \phi v_{f}\right)=0, \\
\phi\left(v_{f}-v_{s}\right)=-k(\phi)\left(\frac{\partial p_{f}}{\partial x}-\rho_{f} g\right), \\
\frac{\partial v_{s}}{\partial x}=-\frac{1}{\xi(\phi)} p_{e}, \quad p_{e}=p_{t o t}-p_{f}, \\
\frac{\partial p_{t o t}}{\partial x}=-\rho_{t o t} g, \quad p_{t o t}=\phi p_{f}+(1-\phi) p_{s}, \quad \rho_{t o t}=\phi \rho_{f}+(1-\phi) \rho_{s} .
\end{gathered}
$$

We seek a solution of this system in the domain $(x, t) \in Q_{T}=\Omega \times(0, T), \Omega=(0,1)$, under the boundary and initial conditions

$$
\left.v_{s}\right|_{x=0, x=1}=\left.v_{f}\right|_{x=0, x=1}=0,\left.\quad \phi\right|_{t=0}=\phi^{0}(x),\left.\quad \rho_{f}\right|_{t=0}=\rho^{0}(x) .
$$

This quasi-linear system of equations describes 1D non-stationary isothermal motion of magma in porous rock. The laws of conservation of mass for each phase, Darcy's law for fluid phase, taking into account the motion of a solid skeleton, the rheological law and the equation of conservation of momentum for system describe this process [1-3]. Here $\rho_{f}, \rho_{s}, v_{f}, v_{s}$ are, respectively, real density and velocity of solid and fluid phases, $\phi$ is the porosity, $p_{f}, p_{s}$ are, respectively, pressures of the fluid and solid phases; $p_{e}$ is the effective pressure, $p_{t o t}$ is the total pressure, $\rho_{t o t}$ is the density of the two-phase medium, $g$ is the density of the mass forces; $k(\phi)$ is the coefficient of filtration, $\xi(\phi)$ is the coefficient of rock shear viscosity (specified function).

\footnotetext{
*papin@math.asu.ru

†tma25@mail.ru

(C) Siberian Federal University. All rights reserved
} 
The problem is written in the Eulerian coordinates $x, t$. The real density of the solid particles $\rho_{s}$ is assumed constant. The unknown quantities are $\phi, \rho_{f}, v_{f}, v_{s} p_{f}, p_{s}$. The system of equations $(1)-(4)$ is closed either by using the equation of state of the fluid phase $p_{f}=p\left(\rho_{f}\right)$ (in particular, the relationship may be, commonly used in applications $\frac{d p_{f}}{d \rho_{f}}=\frac{1}{\beta_{f} \rho_{f}}$, where $\beta_{f}$ is the fluid compressibility [1-3]).

The numerical studies of various initial boundary-value problems for systems of equations (1)(4) were carried out in [2,3]. Some exact solutions have been constructed in [4]. In these studies the following dependencies of the functional parameters of the problem was used: $k(\phi)=\bar{k} \phi^{n} / \mu$, $1 / \xi(\phi)=\phi^{m} / \nu$, where $m \in[0,2], n=3 ; \nu, \mu, \bar{k}$ are positive environment settings [2].

Structurally similar systems of equations was considered in [5-7]. In these studies, based on a number of simplifying assumptions, the original system were reduced to one higher order equation. The local solvability of the Cauchy problem in Sobolev spaces was established in [5]. Travelling wave solutions have been studied in $[6,7]$.

In this paper the unique local solvability of problem (1)-(5) is proved in the case when $g=0$ and $\rho_{f}$ is function of pressure.

On $\Omega$ and $Q_{T}$, let us consider several function spaces, using the notation from [8]. Suppose that $\|\cdot\|_{q, \Omega}$ is the norm on the Lebesgue space $L_{q}(\Omega), q \in[1, \infty]$. For brevity, let $\|\cdot\|_{q}=\|\cdot\|_{q, \Omega}$, $\|\cdot\|=\|\cdot\|_{2, \Omega}$. We also use the Hölder spaces $C^{\alpha}(\Omega), C^{k+\alpha}(\Omega)$, where $k$ is a natural number and $\alpha \in(0,1]$ with norms:

$$
\begin{aligned}
& \|f\|_{C^{\alpha}(\Omega)} \equiv|f|_{\alpha, \Omega}=|f|_{0, \Omega}+H_{x}^{\alpha}(f),|f|_{0, \Omega}=\max _{x \in \bar{\Omega}}|f(x)|, \\
& H_{x}^{\alpha}(f)=\sup _{x_{1}, x_{2} \in \Omega}\left|f\left(x_{1}\right)-f\left(x_{2}\right)\right|\left|x_{1}-x_{2}\right|^{-\alpha}, \\
& \|f\|_{C^{k+\alpha}(\Omega)} \equiv|f|_{k+\alpha, \Omega}=\sum_{m=0}^{k}\left\|D_{x}^{m} f\right\|_{0, \Omega}+H^{\alpha}\left(D_{x}^{k} f\right) .
\end{aligned}
$$

For functions given on $Q_{T}$, we need the space $C^{k+\alpha, m+\beta}\left(Q_{T}\right)$, where $k, m$ are natural numbers and $(\alpha, \beta) \in(0,1]$, with norm $\|f\|_{C^{k+\alpha, m+\beta}\left(Q_{T}\right)} \equiv|f|_{k+\alpha, m+\beta, Q_{T}}=\sum_{l=0}^{k}\left\|D_{x}^{l} f\right\|_{0, Q_{T}}+$ $\sum_{j=1}^{m}\left\|D_{t}^{j} f\right\|_{0, Q_{T}}+H_{x}^{\alpha}\left(D_{x}^{k} f\right)++H_{t}^{\beta}\left(D_{x}^{k} f\right)+H_{x}^{\alpha}\left(D_{t}^{m} f\right)+H_{t}^{\beta}\left(D_{t}^{m} f\right)$, where

$$
\begin{aligned}
& H_{x}^{\alpha}(f(x, t))=\sup _{x_{1}, x_{2} \in \Omega, t \in(0, T)}\left|f\left(x_{1}, t\right)-f\left(x_{2}, t\right)\right|\left|x_{1}-x_{2}\right|^{-\alpha}, \\
& H_{t}^{\beta}(f(x, t))=\sup _{t_{1}, t_{2} \in(0, T), x \in \Omega}\left|f\left(x, t_{1}\right)-f\left(x, t_{2}\right)\right|\left|t_{1}-t_{2}\right|^{-\beta} .
\end{aligned}
$$

In the case $k=m$ and $\alpha=\beta$, we use the notation $C^{k+\alpha}\left(Q_{T}\right)$.

In this paper by a solution of problem (1)-(5) we mean the set of functions $v_{s} \in$ $C^{3+\alpha, 1+\alpha / 2}\left(Q_{T}\right)\left(\phi, \rho_{f}, p_{f}, p_{s}\right) \in C^{2+\alpha, 1+\alpha / 2}\left(Q_{T}\right), v_{f} \in C^{1+\alpha, 1+\alpha / 2}\left(Q_{T}\right)$, such that $0<\phi<1$, $\rho_{f}>0, p_{f}>0$. These functions satisfy the equations (1)-(4) and the initial and boundary conditions (5) and regarded as continuous functions in $\bar{Q}_{T}$.

Let us state the main results of the paper.

Theorem 1. Suppose that $g=0$ and the data of problem (1)-(5) satisfies the following conditions:

1) the functions $k(\phi), \xi(\phi), p_{f}\left(\rho_{f}\right)$ and their derivatives up to the second order are continuous for $\phi \in(0,1), \rho_{f}>0$, and satisfy the conditions

$$
\begin{gathered}
k_{0}^{-1} \phi^{q_{1}}(1-\phi)^{q_{2}} \leqslant k(\phi) \leqslant k_{0} \phi^{q_{3}}(1-\phi)^{q_{4}}, \quad 1 / \xi(\phi)=a_{0}(\phi) \phi^{\alpha_{1}}(1-\phi)^{\alpha_{2}-1}, \quad 0<R_{1} \leqslant a_{0}(\phi) \leqslant R_{2}, \\
k_{0}^{-1} \rho_{f}^{q_{5}} \leqslant p_{f}\left(\rho_{f}\right) \leqslant k_{0} \rho_{f}^{q_{6}}, \quad k_{0}^{-1} \rho_{f}^{q_{7}} \leqslant \frac{\partial p_{f}\left(\rho_{f}\right)}{\partial \rho_{f}} \leqslant k_{0} \rho_{f}^{q_{8}},
\end{gathered}
$$


where $k_{0}, \alpha_{i}, R_{i}, i=1,2$ are positive constants, $q_{1}, \ldots, q_{8}$ are fixed real parameters;

2) the initial functions $\phi^{0}, \rho^{0}$ satisfy the following smoothness conditions: $\phi^{0} \in C^{2+\alpha}(\bar{\Omega}), \rho^{0} \in$ $C^{2+\alpha}(\bar{\Omega})$ and the matching conditions

$$
\left.\frac{d p_{f}\left(\rho^{0}\right)}{d x}\right|_{x=0, x=1}=0
$$

as well as satisfy the inequalities

$$
0<m_{0} \leqslant \phi^{0}(x) \leqslant M_{0}<1, \quad 0<m_{1} \leqslant \rho^{0}(x) \leqslant M_{1}<\infty, \quad x \in \bar{\Omega},
$$

where $m_{0}, M_{0}, m_{1}, M_{1}$ are given positive constants. Then problem (1)-(5) has a local solution, i.e., there exists a value of $t_{0} \in(0, T)$ such that

$$
\begin{gathered}
v_{s}(x, t) \in C^{3+\alpha, 1+\alpha / 2}\left(\bar{Q}_{t_{0}}\right),\left(\phi(x, t), p_{s}(x, t), p_{f}(x, t), \rho_{f}(x, t)\right) \in C^{2+\alpha, 1+\alpha / 2}\left(\bar{Q}_{t_{0}}\right), \\
v_{f}(x, t) \in C^{1+\alpha, 1+\alpha / 2}\left(\bar{Q}_{t_{0}}\right) . \\
\text { Moreover, } 0<\phi(x, t)<1, \quad \rho_{f}(x, t)>0 \text { в } \bar{Q}_{t_{0}} .
\end{gathered}
$$

\section{Local solvability}

Under the conditions of the theorem into force (4) we have $p_{t o t}=p^{0}(t)$. Following [9], we rewrite the system (1)-(3). Suppose that $\bar{x}=\bar{x}(\tau, x, t)$ is a solution of the Cauchy problem

$$
\frac{\partial \bar{x}}{\partial \tau}=v_{s}(\bar{x}, \tau),\left.\quad \bar{x}\right|_{\tau=t}=x
$$

We set $\hat{x}=\bar{x}(0, x, t)$ and take $\hat{x}$ and $t$ for the new variables. Then $1-\phi(\hat{x}, t)=\left(1-\phi^{0}(\hat{x})\right) \hat{J}(\hat{x}, t)$, where $\hat{J}(\hat{x}, t)=\frac{\partial \hat{x}}{\partial x}(\hat{x}, t)$ is the Jacobian of the transformation. The system of equations $(1)-(3)$ in the new variables is of the form

$$
\begin{aligned}
\frac{\partial(1-\hat{\phi})}{\partial t}+\frac{(1-\hat{\phi})^{2}}{1-\phi^{0}} \frac{\partial \hat{v}_{s}}{\partial \hat{x}} & =0, \quad \frac{\partial}{\partial t}\left(\hat{\rho}_{f} \hat{\phi}\right)+\frac{(1-\hat{\phi})}{1-\phi^{0}} \frac{\partial}{\partial \hat{x}}\left(\hat{\rho}_{f} \hat{\phi}_{f}\right)=v_{s} \frac{(1-\hat{\phi})}{1-\phi^{0}} \frac{\partial}{\partial \hat{x}}\left(\hat{\rho}_{f} \hat{\phi}\right) \\
\hat{\phi}\left(\hat{v}_{s}-\hat{v}_{f}\right) & =k(\hat{\phi}) \frac{(1-\hat{\phi})}{1-\phi^{0}} \frac{\partial \hat{p}_{f}}{\partial \hat{x}}, \quad \frac{(1-\hat{\phi})}{1-\phi^{0}} \frac{\partial \hat{v}_{s}}{\partial \hat{x}}=-a_{1}(\hat{\phi}) \hat{p}_{e}
\end{aligned}
$$

where $a_{1}(\phi)=1 / \xi(\phi)$.

Since

$$
v_{s} \frac{\partial}{\partial \hat{x}}\left(\hat{\rho}_{f} \hat{\phi}\right)=\frac{\partial}{\partial \hat{x}}\left(\hat{\rho}_{f} \hat{\phi} v_{s}\right)-\hat{\rho}_{f} \hat{\phi} \frac{\partial v_{s}}{\partial \hat{x}},
$$

it follows that the continuity equation for the liquid phase can be reduced to the form

$$
\frac{1}{(1-\hat{\phi})} \frac{\partial}{\partial t}\left(\hat{\rho}_{f} \hat{\phi}\right)+\frac{1}{1-\phi^{0}} \frac{\partial}{\partial \hat{x}}\left(\hat{\rho}_{f} \hat{\phi}\left(\hat{v}_{f}-v_{s}\right)\right)+\frac{1}{1-\phi^{0}} \hat{\rho}_{f} \hat{\phi} \frac{\partial v_{s}}{\partial \hat{x}}=0 \text {. }
$$

Using the continuity equation for the solid phase, we find that

$$
\frac{\partial}{\partial t}\left(\hat{\rho}_{f} \frac{\hat{\phi}}{1-\hat{\phi}}\right)+\frac{1}{\left(1-\phi^{0}\right)} \frac{\partial}{\partial \hat{x}}\left(\hat{\rho}_{f} \hat{\phi}\left(\hat{v}_{f}-\hat{v}_{s}\right)\right)=0
$$

Finally, passing from $(\hat{x}, t)$ to the mass Lagrangian variables $(y, t)$ by the rule

$$
\left(1-\phi^{0}(\hat{x})\right) d \hat{x}=d y, \quad y(\hat{x})=\int_{0}^{\hat{x}}\left(1-\phi^{0}(\eta)\right) d \eta \in[0,1]
$$


and preserving the notation $y$ for the variable $x$, we obtain

$$
\begin{gathered}
\frac{\partial(1-\phi)}{\partial t}+(1-\phi)^{2} \frac{\partial v_{s}}{\partial x}=0, \quad \frac{\partial}{\partial t}\left(\rho_{f} \frac{\phi}{1-\phi}\right)+\frac{\partial}{\partial x}\left(\rho_{f} \phi\left(v_{f}-v_{s}\right)\right)=0 \\
\phi\left(v_{s}-v_{f}\right)=k(\phi)(1-\phi) \frac{\partial p_{f}}{\partial x} \\
(1-\phi) \frac{\partial v_{s}}{\partial x}=-a_{1}(\phi) p_{e}, \quad p_{e}=p^{0}(t)-p_{f} .
\end{gathered}
$$

Finally, we turn to the dimensionless variables

$$
\begin{gathered}
t^{\prime}=\frac{t}{t_{1}}, \quad x^{\prime}=\frac{x}{L}, \quad v_{s}^{\prime}=\frac{v_{s}}{v_{1}}, \quad v_{f}^{\prime}=\frac{v_{f}}{v_{1}}, \quad \rho_{f}^{\prime}=\frac{\rho_{f}}{\rho_{s}}, \\
p_{f}^{\prime}=\frac{p_{f}}{p_{1}}, \quad p_{s}^{\prime}=\frac{p_{s}}{p_{1}}, \quad p_{e}^{\prime}=\frac{p_{e}}{p_{1}}, \quad p_{t o t}^{\prime}=\frac{p_{t o t}}{p_{1}}, \quad a_{1}^{\prime}(\phi)=\frac{a_{1}(\phi)}{a^{0}}, \quad k^{\prime}(\phi)=\frac{k(\phi)}{k_{1}},
\end{gathered}
$$

where $L=\int_{0}^{1}\left(1-\phi^{0}(\eta)\right) d \eta, t_{1}=\frac{L}{v_{1}}, a^{0}=\frac{v_{1}}{L p_{1}}, k_{1}=\frac{v_{1} L}{p_{1}}, v_{1}, p_{1}$ are fixed positive quantities having the dimension of velocity and pressure accordingly.

Then the domain $x^{\prime}$ is the unit interval $[0,1]$ and the system of equations will retain its structure (dashes omitted).

Using the rheological relationship, Darcy's law and the conditions $\left.v_{s}\right|_{x=0,1}=0$, we find that

$$
p^{0}(t)=\int_{0}^{1} \frac{a_{1}(\phi)}{1-\phi} p_{f} d x\left(\int_{0}^{1} \frac{a_{1}(\phi)}{1-\phi} d x\right)^{-1} \equiv P^{0}\left(\phi, \rho_{f}\right) .
$$

Taking into account Darcy's law, the second equation of the system assumes the form

$$
\frac{\partial}{\partial t}\left(\rho_{f} \frac{\phi}{1-\phi}\right)-\frac{\partial}{\partial x}\left(\rho_{f} k(\phi)(1-\phi) \frac{\partial p_{f}}{\partial x}\right)=0 .
$$

From the first and fourth equations of the system follows that

$$
\frac{1}{1-\phi} \frac{\partial \phi}{\partial t}=a_{1}(\phi)\left(p_{f}-p^{0}\right)
$$

This equation can be written as

$$
\frac{\partial G(\phi)}{\partial t}=p_{f}-p^{0}
$$

where the function $G(\phi)$ is defined by the equation

$$
\frac{d G(\phi)}{d \phi}=\frac{1}{(1-\phi) a_{1}(\phi)}
$$

Let

$$
a(\phi)=\frac{\phi}{1-\phi}, \quad K(\phi)=k(\phi)(1-\phi), \quad b\left(\rho_{f}\right)=\rho_{f} \frac{\partial p_{f}\left(\rho_{f}\right)}{\partial \rho_{f}} .
$$

Taking into account the conditions (5), we obtain the following problem for finding functions $\rho_{f}, \phi$ :

$$
\begin{gathered}
\frac{\partial}{\partial t}\left(a(\phi) \rho_{f}\right)-\frac{\partial}{\partial x}\left(K(\phi) b\left(\rho_{f}\right) \frac{\partial \rho_{f}}{\partial x}\right)=0, \\
\frac{\partial G(\phi)}{\partial t}=p_{f}\left(\rho_{f}\right)-p^{0}(t), \\
\left.\frac{\partial \rho_{f}}{\partial x}\right|_{x=0, x=1}=0,\left.\quad \rho_{f}\right|_{t=0}=\rho^{0}(x),\left.\quad \phi\right|_{t=0}=\phi^{0}(x) .
\end{gathered}
$$


Lemma 1. Let the data of problem (6)-(8) satisfy the conditions of the theorem. Then problem (6)-(8) has a unique local solution, i.e., there exists a value of $t_{0}$ such that

$$
\left(\phi(x, t), \rho_{f}(x, t)\right) \in C^{2+\alpha, 1+\alpha / 2}\left(\bar{Q}_{t_{0}}\right) .
$$

Furthermore, $0<\phi(x, t)<1, \rho_{f}(x, t)>0$ in $\bar{Q}_{t_{0}}$.

The solvability of problem (6)-(8) is established by using the Tikhonov- Schauder fixed-point theorem: if $V$ is a compact convex closed set of Banach space $B$ and the operator $\Lambda$ maps $V$ into itself continuously in the norm of $B$, then there is a fixed point on $V$ [10, pp. 227].

Since the function $\psi=G(\phi)$ is strictly monotone, at $\phi \in(0,1)$, that the inverse function is exist: $\phi=G^{-1}(\psi)$. Assuming that $\rho(x, t)=\rho_{f}(x, t)-\rho^{0}(x), \quad \omega(x, t)=G(\phi)-G\left(\phi^{0}\right)$. We represent the equations $(6),(7)$ in the form

$$
\begin{aligned}
\frac{\partial}{\partial t}\left(a(\omega)\left(\rho+\rho^{0}\right)\right) & =\frac{\partial}{\partial x}\left(K(\omega) b\left(\rho+\rho^{0}\right) \frac{\partial\left(\rho+\rho^{0}\right)}{\partial x}\right), \\
\frac{\partial \omega}{\partial t} & =p_{f}\left(\rho+\rho^{0}\right)-p^{0}(t) .
\end{aligned}
$$

Here $a(\omega)=\frac{\phi(\omega)}{1-\phi(\omega)}, K(\omega)=k(\phi(\omega))(1-\phi(\omega)), \phi(\omega)=G^{-1}\left(\omega+G\left(\phi^{0}\right)\right)$. Moreover,

$$
\left.\rho\right|_{t=0}=\left.\omega\right|_{t=0}=\left.\frac{\partial\left(\rho+\rho^{0}\right)}{\partial x}\right|_{x=0, x=1}=0
$$

For the Banach space, we choose the space $C^{2+\beta, 1+\beta / 2}\left(\bar{Q}_{t_{0}}\right)$, where $\beta$ is any number from the interval $(0, \alpha), \alpha \in[0,1)$. Let

$$
\begin{gathered}
V=\left\{\bar{\rho}(x, t), \bar{\omega}(x, t) \in C^{2+\alpha, 1+\alpha / 2}\left(\bar{Q}_{t_{0}}\right)|\quad \bar{\rho}|_{t=0}=\left.\bar{\omega}\right|_{t=0}=\left.\frac{\partial \bar{\rho}}{\partial x}\right|_{x=0, x=1}=0,\right. \\
\frac{m_{1}}{2}-\rho^{0}(x) \leqslant \bar{\rho}(x, t) \leqslant 2 M_{1}-\rho^{0}(x)<\infty, \\
G\left(m_{0} / 2\right)-G\left(\phi^{0}\right) \leqslant \bar{\omega}(x, t) \leqslant G\left(\frac{M_{0}+1}{2}\right)-G\left(\phi^{0}\right)<\infty, \quad(x, t) \in Q_{t_{0}}, \\
\left.\left(|\bar{\omega}|_{1+\alpha,(1+\alpha) / 2, Q_{t_{0}}},|\bar{\rho}|_{1+\alpha,(1+\alpha) / 2, Q_{t_{0}}}\right) \leqslant K_{1},\left(|\bar{\omega}|_{2+\alpha,(2+\alpha) / 2, Q_{t_{0}}},|\bar{\rho}|_{2+\alpha,(2+\alpha) / 2, Q_{t_{0}}}\right) \leqslant K_{1}+K_{2}\right\},
\end{gathered}
$$

where $K_{1}$ is an arbitrary positive constant, while the positive constant $K_{2}$ will be given later. We note that on the set $V$ following inequalities hold: $0<\frac{m_{0}}{2} \leqslant \phi(\bar{\omega}) \leqslant \frac{M_{0}+1}{2}<1, a(\bar{\omega})>0$, $K(\bar{\omega})>0$.

Let us construct an operator $\Lambda$ mapping $V$ in $V$. Suppose that $\bar{\omega}, \bar{\rho} \in V$. Using (10), we define the function $\omega$ by the equality

$$
\omega=\int_{0}^{t}\left(p_{f}\left(\bar{\rho}(x, \tau)+\rho^{0}(x)\right)-\int_{0}^{1} \frac{a_{1}(\phi(\bar{\omega}))}{1-\phi(\bar{\omega})} p_{f}\left(\bar{\rho}(x, \tau)+\rho^{0}(x)\right) d x\left(\int_{0}^{1} \frac{a_{1}(\phi(\bar{\omega}))}{1-\phi(\bar{\omega})} d x\right)^{-1}\right) d \tau .
$$

From the representation (12) it follows that smoothness $\omega$ is determined by the smoothness of functions $\bar{\rho}, \rho^{0}$ and $p^{0}$. In particular, we have an estimate

$$
|\omega|_{2+\alpha, 1+\alpha / 2, Q_{t_{0}}}=C_{1}\left(m_{0}, M_{0}, m_{1}, M_{1}, K_{1}, T,\left|\rho^{0}\right|_{2+\alpha, \Omega}\right)\left(1+t_{0}\left|\bar{\rho}_{x x}\right|_{\alpha, \alpha / 2, \Omega}\right) .
$$


Lemma 2. Let function $a_{1}(\phi), \phi \in(0,1)$ satisfies the following condition

$$
(1-\phi) a_{1}(\phi)=a_{0}(\phi) \phi^{\alpha_{1}}(1-\phi)^{\alpha_{2}}, \quad 0<R_{1} \leqslant a_{0}(\phi) \leqslant R_{2},
$$

where $R_{i}>0, \alpha_{i}>0, i=1,2$. Then we have the estimate of the form

$$
R_{2}\left|G\left(\phi_{1}\right)-G\left(\phi_{2}\right)\right| \geqslant\left|\phi_{1}-\phi_{2}\right| .
$$

Proof. Assume without loss of generality that $0<\phi_{1} \leqslant \phi_{2}<1$. From the definition of functions $G(\phi)$ and $a_{1}(\phi)$, we have

$$
0<\Delta G \equiv G\left(\phi_{2}\right)-G\left(\phi_{1}\right)=\int_{\phi_{1}}^{\phi_{2}} \frac{d s}{(1-s) a_{1}(s)} \geqslant \frac{1}{R_{2}}\left(\phi_{2}-\phi_{1}\right) .
$$

Lemma 2 is proved.

In this way, we have estimate

$$
\left|\phi(x, t)-\phi^{0}(x)\right| \leqslant \delta(t), \quad \delta(t) \longrightarrow 0 \quad \text { as } \quad t \longrightarrow 0,
$$

which implies, that there exists a value $t_{1}=t_{1}\left(m_{0}, M_{0}, m_{1}, M_{1}\right)$, such that for all $t_{0} \leqslant t_{1}$ the following inequality holds

$$
0<\frac{m_{0}}{2} \leqslant \phi(x, t) \leqslant \frac{M_{0}+1}{2}, \quad(x, t) \in Q_{t_{0}} .
$$

Taking into account (13) we also have the estimate for function $\omega(x, t): G\left(\frac{m_{0}}{2}\right) \leqslant \omega(x, t)+$ $G\left(\phi^{0}\right) \leqslant G\left(\frac{M_{0}+1}{2}\right)$.

Using (9), (11) and $\bar{\omega}(x, t)$ we find the function $\rho(x, t)$ as a solution of the problem (here and elsewhere, we assume that the initial and boundary conditions are matched):

$$
\begin{gathered}
\frac{\partial}{\partial t}\left(a(\bar{\omega})\left(\rho+\rho^{0}\right)\right)=\frac{\partial}{\partial x}\left(K(\bar{\omega}) b(\bar{\rho}) \frac{\partial\left(\rho+\rho^{0}\right)}{\partial x}\right) \\
\left.\rho\right|_{t=0}=\left.\frac{\partial \rho_{f}}{\partial x}\right|_{x=0, x=1}=0,\left.\quad \frac{\partial \rho^{0}}{\partial x}\right|_{x=0, x=1}=0 .
\end{gathered}
$$

The equation for $\rho(x, t)$ is uniformly parabolic. In view of the properties of $\bar{\omega}(x, t)$ and $\rho^{0}(x)$ problem (14) has a classical solution [8]. In addition, we have the following estimate:

$$
\left|\frac{1}{a(\bar{\omega})} \frac{\partial a(\bar{\omega})}{\partial t}\right| \leqslant C_{0}\left(m_{0}, M_{0}, m_{1}, M_{1}, \max _{0 \leqslant t \leqslant T}\left|p^{0}(t)\right|\right) .
$$

Under the additional condition smallness for the value of the time interval the following statement holds [9].

Lemma 3. For $t_{0} \leqslant \min \left(t_{1}, t_{2}\right), \quad t_{2}=\ln 2 / C_{0}\left(m_{0}, M_{0}, m_{1}, M_{1}\right)$, the classical solution of problem (14) satisfies the following inequality in $Q_{t_{0}}$ :

$$
0<\frac{m_{1}}{2} \leqslant \rho(x, t)+\rho^{0}(x) \leqslant 2 M_{1}<\infty .
$$


Proof. Further, setting $U(x, t)=\rho(x, t)+\rho^{0}(x)$, we can express problem (14) in the form

$$
\frac{\partial}{\partial t}(a(\bar{\omega}) U)=\frac{\partial}{\partial x}\left(K(\bar{\omega}) b(\bar{\rho}) \frac{\partial U}{\partial x}\right),\left.\quad \frac{\partial U}{\partial x}\right|_{x=0, x=1}=0,\left.\quad U\right|_{t=0}=\rho^{0} .
$$

First, we show that $U(x, t) \geqslant 0,(x, t) \in Q_{t_{0}}$. In equation (15), let us make the change $U(x, t)=$ $-z(x, t)$. Then

$$
z \frac{\partial a}{\partial t}+a \frac{\partial z}{\partial t}=\frac{\partial}{\partial x}\left(K b \frac{\partial z}{\partial x}\right) .
$$

Let

$$
\begin{gathered}
z^{(0)}(x, t)=\max \{z, 0\},\left.\quad z^{(0)}(x, t)\right|_{t=0}=\max \left\{-\rho^{0}, 0\right\}=0, \\
\sigma_{\varepsilon}(x, t)=z^{(0)}(x, t)\left(\left|z^{(0)}(x, t)\right|^{2}+\varepsilon\right)^{-1 / 2}, \varepsilon>0 .
\end{gathered}
$$

Let us multiply the equation for the function $z$ by $\sigma_{\varepsilon}$ and then integrate over $\Omega$. We obtain the equality

$$
\begin{gathered}
\frac{d}{d t} \int_{0}^{1} a\left(\left|z^{(0)}\right|^{2}+\varepsilon\right)^{1 / 2} d x+\int_{0}^{1} \frac{\partial a}{\partial t}\left(z \sigma_{\varepsilon}-\left(\left|z^{(0)}\right|^{2}+\varepsilon\right)^{1 / 2}\right) d x+ \\
+\varepsilon \int_{0}^{1} K b \frac{\partial z}{\partial x} \frac{\partial z^{(0)}}{\partial x}\left(\left|z^{(0)}\right|^{2}+\varepsilon\right)^{-3 / 2} d x=0 .
\end{gathered}
$$

Let $A^{+}(t)=\{x \in \Omega \mid z(x, t)>0\}, \quad A^{-}(t)=\{x \in \Omega \mid z(x, t) \leqslant 0\}$. Then

$$
\begin{gathered}
\int_{0}^{1} \frac{\partial a}{\partial t}\left(z \sigma_{\varepsilon}-\left(\left|z^{(0)}\right|^{2}+\varepsilon\right)^{1 / 2}\right) d x=-\varepsilon \int_{A^{+}(t)} \frac{\partial a}{\partial t}\left(|z|^{2}+\varepsilon\right)^{-1 / 2} d x-\varepsilon^{1 / 2} \int_{A^{-}(t)} \frac{\partial a}{\partial t} d x, \\
\int_{0}^{1} a\left(\left|z^{(0)}\right|^{2}+\varepsilon\right)^{1 / 2} d x=\int_{A^{+}(t)} a\left(|z|^{2}+\varepsilon\right)^{1 / 2} d x+\varepsilon^{1 / 2} \int_{A^{-}(t)} a d x, \\
\left.\int_{0}^{1} a\left(\left|z^{(0)}\right|^{2}+\varepsilon\right)^{1 / 2}\right|_{t=0} d x=\left.\varepsilon^{1 / 2} \int_{0}^{1} a\right|_{t=0} d x, \int_{A^{+}(t)} a\left(|z|^{2}+\varepsilon\right)^{1 / 2} d x \geqslant \int_{A^{+}(t)} a|z| d x=\int_{0}^{1} a z^{(0)} d x .
\end{gathered}
$$

Integrating relation (16) with respect to time, we obtain

$$
\begin{aligned}
& \int_{A^{+}(t)} a\left(|z|^{2}+\varepsilon\right)^{1 / 2} d x+\varepsilon^{1 / 2} \int_{A^{-}(t)} a d x+\varepsilon \int_{0}^{t} \int_{A^{+}(\tau)} K b\left|\frac{\partial z}{\partial x}\right|^{2}\left(z^{2}+\varepsilon\right)^{-3 / 2} d x d \tau= \\
= & \varepsilon \int_{0}^{t} \int_{A^{+}(\tau)} \frac{\partial a}{\partial \tau}\left(|z|^{2}+\varepsilon\right)^{-1 / 2} d x d \tau+\varepsilon^{1 / 2} \int_{0}^{t} \int_{A^{-}(\tau)} \frac{\partial a}{\partial \tau} d x d \tau+\left.\varepsilon^{1 / 2} \int_{0}^{1} a\right|_{t=0} d x .
\end{aligned}
$$

Therefore,

$$
\int_{0}^{1} a z^{(0)} d x \leqslant \varepsilon^{1 / 2} \int_{0}^{t} \int_{0}^{1}\left|\frac{\partial a}{\partial \tau}\right| d x d \tau+\left.\varepsilon^{1 / 2} \int_{0}^{1} a\right|_{t=0} d x
$$

Passing to the limit as $\varepsilon \rightarrow 0$, we find that $z^{(0)}=0$, i.e. $U \geqslant 0$.

After multiplication by $U^{l-1}(x, t), l>2$, equation (15) can be expressed as

$$
\frac{1}{l} \frac{\partial\left(a U^{l}\right)}{\partial t}+(l-1) K b U^{l-2}\left(\frac{\partial U}{\partial x}\right)^{2}+\frac{l-1}{l} U^{l} \frac{\partial a}{\partial t}=\frac{\partial}{\partial x}\left(K b U^{l-1} \frac{\partial U}{\partial x}\right) .
$$


Then

$$
\frac{1}{l} \frac{d}{d t} \int_{0}^{1} a U^{l} d x \leqslant \frac{l-1}{l} \max _{0 \leqslant x \leqslant 1}\left|\frac{1}{a} \frac{\partial a}{\partial t}\right| \int_{0}^{1} a U^{l} d x
$$

Therefore,

$$
\begin{gathered}
y^{\prime}(t) \leqslant \frac{l-1}{l} \max _{0 \leqslant x \leqslant 1}\left|\frac{1}{a} \frac{\partial a}{\partial t}\right| y(t), \quad y^{l}(t)=\int_{0}^{1}\left(a^{1 / l} U\right)^{l} d x, \\
y(t) \leqslant y(0) \exp \left\{\frac{l-1}{l} \int_{0}^{t} \max _{0 \leqslant x \leqslant 1}\left|\frac{1}{a} \frac{\partial a}{\partial t}\right| d \tau\right\} .
\end{gathered}
$$

After passing to the limit as $l \rightarrow \infty$, we obtain

$$
\max _{0 \leqslant x \leqslant 1} U(x, t) \leqslant \max _{0 \leqslant x \leqslant 1} \rho^{0}(x) \exp \left\{\int_{0}^{t} \max _{0 \leqslant x \leqslant 1}\left|\frac{1}{a} \frac{\partial a}{\partial t}\right| d \tau\right\} .
$$

Taking into account the inequality $\max _{0 \leqslant x \leqslant 1} \rho^{0}(x) \leqslant M_{1}$ and choosing $t$ from the condition

$$
t \leqslant t_{2}, \quad \exp \left\{\int_{0}^{t_{2}} \max _{0 \leqslant x \leqslant 1}\left|\frac{1}{a} \frac{\partial a}{\partial t}\right| d \tau\right\} \leqslant 2,
$$

we obtain upper bound for $\rho$. To obtain a lower estimate we represent equation (15) in the form $(z(x, t)=1 / U(x, t))$

$$
\frac{1}{l} \frac{\partial\left(a z^{l}\right)}{\partial t}+(l+1) K b z^{l-2}\left(\frac{\partial z}{\partial x}\right)^{2}-\frac{l+1}{l} z^{l} \frac{\partial a}{\partial t}=\frac{\partial}{\partial x}\left(K b z^{l-1} \frac{\partial z}{\partial x}\right) .
$$

Then we obtain inequality

$$
\frac{1}{l} \frac{d}{d t} \int_{0}^{1} a z^{l} d x \leqslant \frac{l+1}{l} \max _{0 \leqslant x \leqslant 1}\left|\frac{1}{a} \frac{\partial a}{\partial t}\right| \int_{0}^{1} a z^{l} d x
$$

then we obtain the estimate

$$
\max _{0 \leqslant x \leqslant 1} \frac{1}{U(x, t)} \leqslant \max _{0 \leqslant x \leqslant 1} \frac{1}{\rho^{0}(x)} \exp \left\{\int_{0}^{t} \max _{0 \leqslant x \leqslant 1}\left|\frac{1}{a} \frac{\partial a}{\partial t}\right| d \tau\right\} \leqslant \frac{2}{m_{1}} .
$$

Lemma 3 is proved.

In view of Lemma 3 and the properties of $\bar{\omega}$, we have the following estimates [8, Sec. 3]:

$$
\begin{gathered}
|\rho|_{\alpha, \alpha / 2, Q_{t_{0}}} \leqslant C_{2}, \\
|\rho|_{2+\alpha, 1+\alpha / 2, Q_{t_{0}}} \leqslant C_{3}\left(1+\left|\rho^{0}\right|_{2+\alpha, \Omega}+\left|\bar{\rho}_{x}\right|_{\alpha, \alpha / 2, Q_{t_{0}}}+\left|\bar{\omega}_{t}\right|_{\alpha, \alpha / 2, Q_{t_{0}}}+\left|\bar{\omega}_{x}\right|_{\alpha, \alpha / 2, Q_{t_{0}}}\right),
\end{gathered}
$$

in which the constant $C_{2}, C_{3}$ depends on $K_{1}, m_{0}, m_{1}, M_{0}, M_{1}$. Therefore

$$
|\rho|_{2+\alpha, 1+\alpha / 2, Q_{t_{0}}} \leqslant C_{4}\left(K_{1}, m_{0}, m_{1}, M_{0}, M_{1}\right) .
$$

Let $C_{5}=\max \left\{C_{1}, C_{4}\right\}$. Choose $K_{2}$ so that $C_{5} \leqslant \frac{K_{1}+K_{2}}{2}$. Then, for $t_{0}<\min \left(t_{1}, t_{2},\left(K_{1}+\right.\right.$ $\left.K_{2}\right)^{-1}$ ) we obtain

$$
|\rho|_{2+\alpha, 1+\alpha / 2, Q_{t_{0}}} \leqslant K_{1}+K_{2}, \quad|\omega|_{2+\alpha, 1+\alpha / 2, Q_{t_{0}}} \leqslant K_{1}+K_{2}
$$


It remains to verify conditions

$$
|\rho|_{1+\alpha,(1+\alpha) / 2, Q_{t_{0}}} \leqslant K_{1}, \quad|\omega|_{1+\alpha,(1+\alpha) / 2, Q_{t_{0}}} \leqslant K_{1} .
$$

Integrating equation (14) with respect to time, we obtain $|\rho|_{0, Q_{t_{0}}} \leqslant C_{6} t_{0}$. From the equation (12) we obtain $|\omega|_{0, Q_{t_{0}}} \leqslant C_{7} t_{0}$. Further, using for $\rho, \omega$ an inequality of the form [11, pp. 35]

$$
|u|_{1+\alpha,(1+\alpha) / 2, Q_{t_{0}}} \leqslant C|u|_{2+\alpha, 1+\alpha / 2, Q_{t_{0}}}^{c}|u|_{0, Q_{t_{0}}}^{1-c}, \quad c=(1+\alpha)(2+\alpha)^{-1},
$$

we find that there exists a sufficiently small value of $t_{0}$, depending on $K_{1}$ and $K_{2}$, such that the required estimates hold: $|\rho|_{1+\alpha,(1+\alpha) / 2, Q_{t_{0}}} \leqslant K_{1},|\omega|_{1+\alpha,(1+\alpha) / 2, Q_{t_{0}}} \leqslant K_{1}$.

Thus, the operator $\Lambda$ maps the set $V$ into itself for sufficiently small values of $t_{0}$. Using the estimates obtained above, we can easily show the continuity of the operator $\Lambda$ in the norm of the space $C^{2+\beta, 1+\beta / 2}\left(\bar{Q}_{t_{0}}\right)$. By the Tikhonov-Schauder theorem, there exists a fixed point $(\rho, \omega) \in V$ of the operator $\Lambda$.

Let us establish uniqueness of the solution of problem (6)-(8).

Suppose that $\left(\rho_{f}^{(1)}, \phi^{(1)}\right)$ and $\left(\rho_{f}^{(2)}, \phi^{(2)}\right)$ are two different solutions of problem. Their difference $\rho=\rho_{f}^{(1)}-\rho_{f}^{(2)}, \phi=\phi^{(1)}-\phi^{(2)}$ is the solution of the linear homogeneous system

$$
\begin{gathered}
\frac{\partial}{\partial t}\left(d_{0} \rho+d_{1} \phi\right)-\frac{\partial}{\partial x}\left(d_{2} \frac{\partial \rho}{\partial x}+d_{3} \rho+d_{4} \phi\right)=0, \\
\frac{\partial}{\partial t}\left(h_{0} \phi\right)-h_{1} \rho+V(t)=0, \\
V(t)=P^{0}\left(\phi^{(1)}, \rho_{f}^{(1)}\right)-P^{0}\left(\phi^{(2)}, \rho_{f}^{(2)}\right)=\int_{0}^{1}\left(h_{2}(x, t) \rho(x, t)+h_{3}(x, t) \phi(x, t)\right) d x,
\end{gathered}
$$

with zero initial and boundary conditions $\left.\phi\right|_{t=0}=\left.\rho\right|_{t=0}=\left|\frac{\partial \rho}{\partial x}\right|_{x=0, x=1}=0$.

The coefficients

$$
\begin{aligned}
& d_{0}=a\left(\phi^{(1)}\right)>0, \quad d_{1}=\frac{\left(a\left(\phi^{(1)}\right)-a\left(\phi^{(2)}\right)\right) \rho_{f}^{(2)}}{\phi^{(1)}-\phi^{(2)}}>0, \quad d_{2}=K\left(\phi^{(2)}\right) b\left(\rho_{f}^{(2)}\right)>0, \\
& d_{3}=K\left(\phi^{(1)}\right) \frac{b\left(\rho_{f}^{(1)}\right)-b\left(\rho_{f}^{(2)}\right)}{\rho_{f}^{(1)}-\rho_{f}^{(2)}} \frac{\partial \rho_{f}^{(1)}}{\partial x}, \quad d_{4}=b\left(\rho_{f}^{(2)}\right) \frac{K\left(\phi^{(1)}\right)-K\left(\phi^{(2)}\right)}{\phi^{(1)}-\phi^{(2)}} \frac{\partial \rho_{f}^{(1)}}{\partial x} \\
& h_{0}=\frac{G\left(\phi^{(1)}\right)-G\left(\phi^{(2)}\right)}{\phi^{(1)}-\phi^{(2)}}>0, \quad h_{1}=\frac{p\left(\rho_{f}^{(1)}\right)-p\left(\rho_{f}^{(2)}\right)}{\rho_{f}^{(1)}-\rho_{f}^{(2)}} \\
& h_{2}=\frac{a_{1}\left(\phi^{(1)}\right)}{1-\phi^{(1)}} \frac{p_{f}\left(\rho_{f}^{(1)}\right)-p_{f}\left(\rho_{f}^{(2)}\right)}{\rho_{f}^{(1)}-\rho_{f}^{(2)}}\left(\int_{0}^{1} \frac{a_{1}\left(\phi^{(1)}\right)}{1-\phi^{(1)}} d x\right)^{-1}, \\
& h_{3}=\left(\frac{a_{1}\left(\phi^{(1)}\right)}{1-\phi^{(1)}}-\frac{a_{1}\left(\phi^{(2)}\right)}{1-\phi^{(2)}}\right)\left(\phi^{(1)}-\phi^{(2)}\right)^{-1} \times \\
& \times\left(p_{f}\left(\rho_{f}^{(2)}\right)\left(\int_{0}^{1} \frac{a_{1}\left(\phi^{(1)}\right)}{1-\phi^{(1)}} d x\right)^{-1}-\int_{0}^{1} \frac{a_{1}\left(\phi^{(2)}\right)}{1-\phi^{(2)}} p_{f}\left(\rho_{f}^{(2)}\right) d x\left(\int_{0}^{1} \frac{a_{1}\left(\phi^{(1)}\right)}{1-\phi^{(1)}} d x \int_{0}^{1} \frac{a_{1}\left(\phi^{(2)}\right)}{1-\phi^{(2)}} d x\right)^{-1}\right)
\end{aligned}
$$

are bounded for all $x \in[0,1], t \in[0, T]$. 
Taking into account (18), equation (17) can be represented as

$$
\frac{\partial}{\partial t}\left(d_{0} \rho\right)+\frac{d_{1}}{h_{0}}\left(h_{1} \rho-V(t)\right)+h_{0} \phi \frac{\partial}{\partial t}\left(\frac{d_{1}}{h_{0}}\right)-\frac{\partial}{\partial x}\left(d_{2} \frac{\partial \rho}{\partial x}+d_{3} \rho+d_{4} \phi\right)=0 .
$$

Multiplying the equation (19) by $\rho(x, t)$ and integrating by $x$ from 0 to 1 , we obtain

$$
\frac{d}{d t} \int_{0}^{1} \rho_{1}^{2}(x, t) d x \leqslant C\left(\int_{0}^{1} \rho_{1}^{2}(x, t) d x+\int_{0}^{1} u^{2}(x, t) d x+V^{2}(t)\right)
$$

where $\rho_{1}(x, t)=d_{0}^{1 / 2}|\rho(x, t)|, u(x, t)=h_{0} \phi(x, t)$. Here the constant $C$ depends on $T$ and quantities

$$
\begin{gathered}
\max _{(x, t) \in Q_{T}} \frac{1}{\phi^{(i)}(x, t)}, \quad \max _{(x, t) \in Q_{T}} \frac{1}{1-\phi^{(i)}(x, t)}, \quad \max _{(x, t) \in Q_{T}} \rho_{f}^{(i)}(x, t), \quad \max _{(x, t) \in Q_{T}} \frac{1}{\rho_{f}^{(i)}(x, t)}, \\
\max _{(x, t) \in Q_{T}}\left\|\frac{\partial \phi^{(i)}(x, t)}{\partial t}\right\|, \max _{(x, t) \in Q_{T}}\left\|\frac{\partial \rho_{f}^{(i)}(x, t)}{\partial t}\right\|, \max _{(x, t) \in Q_{T}}\left\|\frac{\partial \rho_{f}^{(i)}(x, t)}{\partial x}\right\|, \quad i=1,2 .
\end{gathered}
$$

For $V(t)$, we also have $V(t) \leqslant C \int_{0}^{1}\left(\rho_{1}(x, t)+|u(x, t)|\right) d x$.

Integrating equation (18) by time and taking into account the estimate for $V(t)$, we obtain

$$
|u(x, t)| \leqslant C \int_{0}^{t}\left(\rho_{1}(x, \tau)+|V(\tau)|\right) d \tau \leqslant C\left(\int_{0}^{t} \rho_{1}(x, \tau) d \tau+\int_{0}^{t} \int_{0}^{1} \rho_{1}(x, \tau) d x d \tau+\int_{0}^{t} \int_{0}^{1}|u(x, \tau)| d x d \tau\right) .
$$

Integrating last inequality by $x$ from 0 to 1 , we obtain Gronwall inequality for function $\int_{0}^{1}|u(x, t)| d x$

$$
\int_{0}^{1}|u(x, t)| d x \leqslant C\left(\int_{0}^{t} \int_{0}^{1} \rho_{1}(x, \tau) d x d \tau+\int_{0}^{t} \int_{0}^{1}|u(x, \tau)| d x d \tau\right)
$$

Therefore

$$
\int_{0}^{1}|u(x, t)| d x \leqslant C \int_{0}^{t} \int_{0}^{1} \rho_{1}(x, \tau) d x d \tau, \quad|V(t)| \leqslant C\left(\int_{0}^{1} \rho_{1}(x, t) d x+\int_{0}^{t} \int_{0}^{1} \rho_{1}(x, \tau) d x d \tau\right),
$$

and consequently $|u(x, t)| \leqslant C\left(\int_{0}^{t} \rho_{1}(x, \tau) d \tau+\int_{0}^{t} \int_{0}^{1} \rho_{1}(x, \tau) d x d \tau\right)$. Hence we obtain from $(20)$ :

$$
\frac{d}{d t}\left\|\rho_{1}(t)\right\|^{2} \leqslant C\left(\left\|\rho_{1}(t)\right\|^{2}+\int_{0}^{t}\left\|\rho_{1}(\tau)\right\|^{2} d \tau\right)
$$

We set $w(t)=\int_{0}^{t}\left\|\rho_{1}(\tau)\right\| d \tau$, then from (21) we obtain $\frac{d^{2} w}{d t^{2}} \leqslant C\left(\frac{d w}{d t}+w(t)\right)$. This yields $\frac{d}{d t}\left(e^{t}\left(\frac{d w}{d t}-(C+1) w\right)\right)+w e^{t} \leqslant 0$, so we have inequality $\frac{d w}{d t} \leqslant(C+1) w$. Therefore $w(t)=0$ и $\rho=0, \phi=0$. Lemma 1 is proved.

After finding $\phi$ and $\rho_{f}$, we find $p_{t o t}=P^{0}\left(\rho_{f}, \phi\right)$. Then we find $p_{s}=\left(p^{0}-\phi p_{f}\right)(1-\phi)^{-1}$. We find $v_{s}$ from the equation $\frac{\partial v_{s}}{\partial x}=-a_{1}(\phi)(1-\phi)^{-1}\left(p_{t o t}-p_{f}\right)$, and from the Darcy's law we obtain $v_{f}=v_{s}-k(\phi)(1-\phi) \phi^{-1} \frac{\partial p_{f}}{\partial x}$. 
Since $\left(\phi, \rho_{f}\right) \in C^{2+\alpha, 1+\frac{\alpha}{2}}\left(Q_{t_{0}}\right)$, then we have: $v_{s} \in C^{3+\alpha, 1+\frac{\alpha}{2}}\left(Q_{t_{0}}\right)$, $v_{f} \in C^{1+\alpha, 1+\frac{\alpha}{2}}\left(Q_{t_{0}}\right),\left(p_{f}, p_{s}\right) \in C^{2+\alpha, 1+\frac{\alpha}{2}}\left(Q_{t_{0}}\right)$.

This work was partially supported by the grants RFBR 16-08-00291 "Hydroelastic and thermodynamic effects with inter-action of poroelastic ice and structures" and state assignment of the Ministry of Education and Science no. 2014/2.

\section{References}

[1] J.Bear, Dynamics of Fluids in Porous Media, Elsevier, New York, 1972.

[2] J.A.D.Connolly, Y.Y.Podladchikov, Compaction-driven fluid flow in viscoelastic rock, Geodin. Acta, 11(1998), 55-84.

[3] C.Morency, R.S.Huismans, C.Beaumont, P.Fullsack, A numerical model for coupled fluid flow and matrix deformation with applications to disequilibrium compaction and delta stability, Journal of Geophysical Research, 112(2007), B10407.

[4] I.G.Akhmerova, A.A.Papin, M.A.Tokareva, Mathematical models of heterogeneous media. Part 1., Altai Gos. Univ., Barnaul, 2012 (in Russian).

[5] M.Simpson, M.Spiegelman, M.I.Weinstein, Degenerate dispersive equations arising in the stady of magma dynamics, Nonlinearity, 20(2007), 21-49.

[6] A.M.Abourabia, K.M.Hassan, A.M.Morad, Analytical solutions of the magma equations for molten rocks in a granular matrix, Chaos Solutions Fract., 42(2009), 1170-1180.

[7] Y.Geng, L.Zhang, Bifurcations of traveling wave solutions for the magma equation, Applied Mathematics and computation, 217(2010), 1741-1748.

[8] O.A.Ladyzhenskaya, V.A.Solonnikov, N.N.Ural'tseva, Linear and Quasilinear Equations of Parabolic Type, Moscow, Nauka, 1967 (in Russian).

[9] A.A.Papin, I.G.Akhmerova, Solvability of the system of equations of one-dimensional motion of a heat-conducting two-phase mixture, Mathematical Notes, 87(2010), no. 2, 230-243.

[10] R.Edwards, Functional Analysis: Theory and Applications, New York, 1965.

[11] S.N.Antontsev, A.V.Kazhikhov, V.N.Monakhov, Boundary-Value Problems of the Mechanics of Inhomogeneous Fluids, Nauka, Sibirskoe Otdelenie, Novosibirsk, 1983 (in Russian).

\section{Локальная разрешимость системы уравнений одномерного движения магмы}

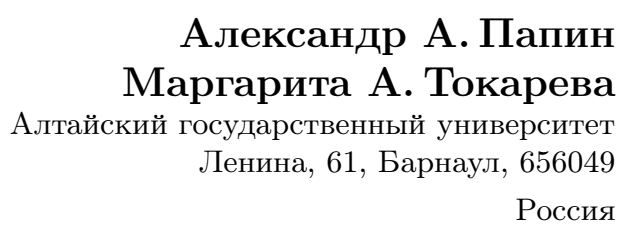

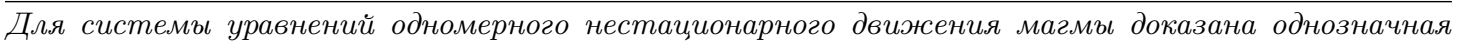
локальная разрешимость начально-краевой задачи.

Ключевые слова: закон Дарси, пороупругость, магма, разрешимость, единственность. 